\title{
A LINEAR HOMOGENIZATION PROBLEM WITH TIME DEPENDENT COEFFICIENT
}

\section{BY}

\author{
MARIA-LUISA MASCARENHAS
}

ABSTRACT. We consider: the homogenization problem

$$
\left\{\begin{array}{l}
(\partial u \varepsilon / \partial t)(x, t)+\beta_{\varepsilon}(x) u_{\varepsilon}(x, t)=0, \quad t \geqslant 0, \\
u_{\varepsilon}(x, 0)=\phi(x),
\end{array}\right.
$$

where $\beta$ is a strictly positive bounded real function, periodic of period 1 , and $\beta_{\varepsilon}(x)=\beta(x / \varepsilon)$; the equivalent integral equation

$$
u_{\varepsilon}(x, t)+\int_{0}^{t} \beta_{\varepsilon}(x) u_{\varepsilon}(x, s) d s=\phi(x) ;
$$

and the homogenized equation

$$
u_{0}(x, t)+\int_{0}^{t} K(t-s) u_{0}(s) d s=\phi(x),
$$

where $K$ is a unique, well-defined function depending on $\beta$. We study this problem for a time dependent $\beta$, and characterize a two-variable function $K(s, t)$ satisfying

$$
u_{0}(x, t)+\int_{0}^{t} K(s, t-s) u_{0}(x, s) d s=\phi(x)
$$

and study its uniqueness.

1. Introduction. In linear homogenization theory it is possible for a differential equation with an integral term (a memory effect) to arise from an equation with pure differential structure: viscoelastic behaviour of composite material is an example (Sanchez-Palencia [3]).

The proofs rely on Laplace transform and standard homogenization techniques in the space variables. Although the convolution kernel is given by a formula, its properties are not easy to derive from it; this method cannot be generalized to time dependent coefficients or to nonlinear problems. This paper is concerned with a simple time dependent problem and studies the properties of the kernel of the integral term (no longer a convolution) arising in the homogenized limit.

Let $\beta(x, t)$ be a real function, periodic in $x$ of period 1 for each $t$ in $[0,+\infty[$ satisfying:

(i) $0<\underline{\alpha} \leqslant \beta(x, t) \leqslant \bar{\alpha}, \quad \forall x \in[0,1], \forall t \in[0,+\infty[$,

(ii) $|\partial \beta(x, t) / \partial t| \leqslant \overline{\bar{\alpha}}, \quad \forall x \in[0,1], \forall t \in[0,+\infty[$.

Received by the editors September 22, 1982.

1980 Mathematics Subject Classification. Primary 45A05.

Key words and phrases. Homogenization, convolution, kernel, integral equation.

(C)1984 American Mathematical Society $0002-9947 / 84 \$ 1.00+\$ .25$ per page 
We consider the parameterized problem for $\varepsilon>0$ :

$$
\left\{\begin{array}{l}
\frac{\partial u_{\varepsilon}}{\partial t}(x, t)+\beta_{\varepsilon}(x, t) u_{\varepsilon}(x, t)=0, \\
u_{\varepsilon}(x, 0)=\phi(x),
\end{array}\right.
$$

with $\beta_{\varepsilon}(x, t)=\beta(x / \varepsilon, t)$, and we search for the homogenized equation.

First we reduce (1.2) to the integral equation

$$
u_{\varepsilon}(x, t)+\int_{0}^{t} \beta_{\varepsilon}(x, s) u_{\varepsilon}(x, s) d s=\phi(x), \quad t \geqslant 0 .
$$

Its solution,

$$
u_{\varepsilon}(x, t)=\phi(x) I\left(\beta_{\varepsilon}, t\right)
$$

where

$$
I(\beta, t)=\exp \left(-\int_{0}^{t} \beta(x, \xi) d \xi\right),
$$

converges weakly in $L^{p}(0,1)$ (for $1<p<+\infty$; in the weak measure sense, if $p=1$; weak $^{*}$, if $\left.p=+\infty\right)$ to its average over $[0,1]$ :

$$
u_{\varepsilon}(x, t) \underset{\varepsilon \rightarrow 0^{+}}{\rightarrow} u_{0}(x, t)=\phi(x) \int_{0}^{1} I(\beta, t) d x .
$$

The question is: What form will the integral $\int_{0}^{t} \beta_{\varepsilon}(x, s) u_{\varepsilon}(x, s) d s$ take as $\varepsilon \rightarrow 0^{+}$, or, equivalently, What will the homogenized integral equation be like?

W.l.o.g. we now consider $\phi(x) \equiv 1 . u_{0}(x, t)$ no longer depends on $x$.

Let us examine the following example.

EXAMPLE. $\beta$ does not depend on $t$. There exists only one function $K$, with support lying in $[0,+\infty[$, satisfying

$$
u_{0}(t)+\int_{0}^{t} K(t-s) u_{0}(s) d s=1, \quad \forall t \geqslant 0 .
$$

So we obtain the homogenized equation in integral form with kernel $K$.

Indeed, if we take

$$
S(t)=\left\{\begin{array}{l}
0, \quad t<0 \\
u_{0}(t)=\int_{0}^{1} e^{-t \beta(x)} d x, \quad t \geqslant 0,
\end{array}\right.
$$

(1.4) will become the convolution equation

$$
S+K * S=\chi_{[0,+\infty]} .
$$

Since $S(0)=1$, by differentiation we obtain the equivalent expression

$$
K-K * g=g, \quad \text { with } g(t)= \begin{cases}0, & t<0 \\ -S^{\prime}(t), & t \geqslant 0 .\end{cases}
$$

Since the inverse, in the convolution sense, of $\left(\delta_{0}-g\right)$ is the sum of the series $\delta_{0}+g+g * g+g * g * g+\cdots$, absolutely convergent in $L_{\mathrm{loc}}^{1}(0,+\infty)\left(\int_{0}^{+\infty}|g|=\right.$ $1)$, it is easy to verify that the unique solution of (1.6) is given by $g+g * g+$ $g * g * g+\cdots$, or, equivalently, $\left(\delta_{0}+K\right)=\left(\delta_{0}-g\right)^{*-1}$. 
We now state our main result,

THEOREM 1.1. Let $\beta(x, t)$ be a real function, periodic in $x$ of period 1 for each $t \in\left[0,+\infty\left[\right.\right.$ satisfying (1.1). Then there exists a kernel $K(s, t)$ defined in $\mathbf{R}^{2}$, with support lying in $[0,+\infty[\times[0,+\infty[$, analytic in $t \in[0,+\infty[$, satisfying

$$
S(t)+\int_{0}^{t} K(s, t-s) S(s) d s=1, \quad \forall t \in[0,+\infty[.
$$

This kernel is the unique solution of the convolution equation

$$
K(s, t)-[K(s, \cdot) * g(s, \cdot)](t)=P(s, t)+g(s, t),
$$

where $S, g$ and $P$ are given by

$$
\begin{gathered}
S(t)=\left\{\begin{array}{l}
0, \quad t<0, \\
\int_{0}^{1} I(\beta, t) d x, \quad t>0,
\end{array}\right. \\
g(s, t)=\left\{\begin{array}{l}
0, \quad s<0 \text { or } t<0, \\
\int_{0}^{1} \beta(x, s) e^{-t \beta(x, s)} d m_{s}(x), \quad s, t \in[0,+\infty[,
\end{array}\right.
\end{gathered}
$$

with

$$
d m_{s}(x)=\frac{1}{S(s)} I(\beta, s) d x
$$

and

$$
P(s, t)=\left\{\begin{array}{l}
0, \quad s<0 \text { or } t<0 \\
-\frac{1}{S(s)} \int_{0}^{s} \frac{\partial K}{\partial t}(\xi, t+s-\xi) S(\xi) d \xi, \quad s, t \in[0,+\infty[
\end{array}\right.
$$

Furthermore, the following estimate occurs:

$$
\left|\frac{\partial^{m} K}{\partial t^{m}}(s, t)\right| \leqslant 2^{m} \overline{\boldsymbol{\alpha}}^{m+1} e^{C(\underline{\alpha}, \bar{\alpha}, s) \cdot s}, \quad \forall m \geqslant 0, \forall s, t \in[0,+\infty[,
$$

$C(\underline{\alpha}, \bar{\alpha}, s)$ being a positive real value depending only on the bounds of $\beta(c f .(1.1))$ and $s$.

Note. We shall see later that (1.7) does not characterize the kernel and, otherwise, a function $K$ satisfying (1.8) is unique. Indeed (1.8) expresses the natural time dependence between $K$ and $\beta$ : at each instant $s, K(s, t)$ behaves as if $\beta$ should remain time independent, $K$ being affected only by the previous values of $\beta$.

The proof of the theorem ( $\$ 5)$ relies on the fact that for a step function $\beta$ one can find, in each interval where $\beta$ is constant with respect to the time $t$, a kernel for the homogenized equation.

We shall first prove local existence and local uniqueness theorems ( $(5)$.

The main idea is to approach a function $\beta$ satisfying condition (1.1) by step functions ( $\$ 2)$ and study the convergence of the corresponding kernels $(\S \S 4,5)$.

In $\$ 3$ we study the solution of certain convolution equations of type $G-G * g=g$ which are essential for what follows. 
2. Construction of the kernels corresponding to step functions approaching $\beta$. Let $\beta$ be a real function satisfying (1.1). We take a partition of the bounded interval [0,T [ into $n$ equal subintervals. Let $\beta_{n}(x, t)$ be defined as follows:

$$
\beta_{n}(x, t)=\beta(x, \tau), \quad \forall x \in[0,1], \forall t \in[\tau, \tau+T / n[,
$$

where $[\tau, \tau+T / n[$ represents any element of the partition.

We define the corresponding kernel $K_{n}(s, t)$ by induction over the partition indices with the property of being indefinitely differentiable w.r.t. $t \in[0,+\infty[$ and constant w.r.t. $s \in\left[0, T\right.$ [ whenever $\beta_{n}$ is time independent:

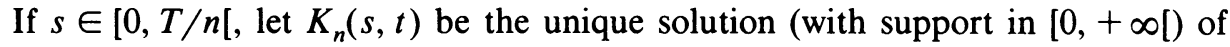
the convolution equation (cf. Example, $\S 1$ ):

$$
G-G * g_{n}(0, \cdot)=g_{n}(0, \cdot), \quad g_{n}(0, t)=\left\{\begin{array}{l}
0, t<0, \\
\int_{0}^{1} \beta_{n}(x, 0) e^{-t \beta_{n}(x, 0)} d x, \quad t \geqslant 0,
\end{array}\right.
$$

which clearly admits derivatives of any order w.r.t $t$.

We suppose the restriction of $K_{n}(s, t)$ to $[\theta, \theta+T / n[\times[0,+\infty[$, having the desired properties of differentiability and constancy, is defined for all $\theta<\tau$.

If $s \in\left[\tau, \tau+T / n\left[\right.\right.$, let $K_{n}(s, t)$ be given by

$$
K_{n}(s, t)=K_{n}(\tau, t)=P_{n}(\tau, t)+\left[P_{n}(\tau, \cdot) * G_{n}(\tau, \cdot)\right](t)+G_{n}(\tau, t),
$$

where $G_{n}(\tau, \cdot)$ is the unique solution (with support in $\left[0,+\infty\left[\right.\right.$ ) of $G-G * g_{n}(\tau, \cdot$ ) $=g_{n}(\tau, \cdot)$, with $g_{n}(\tau, \cdot)$ given by

$$
\begin{gathered}
g_{n}(\tau, t)=\left\{\begin{array}{l}
0, \quad t<0, \\
\int_{0}^{1} \beta_{n}(x, \tau) e^{-t \beta_{n}(x, \tau)} d m_{\tau, n}(x), \quad t \geqslant 0,
\end{array}\right. \\
d m_{\tau, n}(x)=\frac{1}{S_{n}(\tau)} I\left(\beta_{n}, \tau\right) d x,
\end{gathered}
$$

a positive measure such that $\int_{0}^{1} d m_{r, n}(x)=1$,

$$
S_{n}(s)=\int_{0}^{1} I\left(\beta_{n}, s\right) d x
$$

and

$$
P_{n}(\tau, t)=\left\{\begin{array}{l}
0, \quad t<0, \\
-\frac{1}{S_{n}(\tau)} \sum_{0 \leqslant \theta \leqslant \tau-T / n} \int_{\theta}^{\theta+T / n} \frac{\partial K_{n}}{\partial t}(\theta, t+\tau-\xi) S_{n}(\xi) d \xi, \quad t \geqslant 0 .
\end{array}\right.
$$

Obviously $K_{n}(\tau, t)$ is indefinitely differentiable with respect to $t$.

Since $G_{n}(\tau, \cdot)$ is such that $\delta_{0}+G_{n}=\left(\delta_{0}-g_{n}\right)^{*-1}$ (cf. Example, $\S 1$ ), we may say that, for $s \in[\tau, \tau+T / n[, K(s, t)$ is the unique solution of the convolution equation

$$
K_{n}(\tau, t)-\left[K_{n}(\tau, \cdot) * g_{n}(\tau, \cdot)\right](t)=P_{n}(\tau, t)+g_{n}(\tau, t) .
$$


The function $K_{n}$ is thus defined for all $s \in[0, T[$ and $t \in \mathbf{R}$, vanishing for $t<0$. Setting $g_{n}(s, t)=g_{n}(\tau, t)$ and $P_{n}(s, t)=P_{n}(\tau, t)$ for $s \in[\tau, \tau+T / n[$, we have, for all $(s, t) \in[0, T[\times[0,+\infty[$,

$$
K_{n}(s, t)-\left[K_{n}(s, \cdot) * g_{n}(s, \cdot)\right](t)=P_{n}(s, t)+g_{n}(s, t) .
$$

In particular, for $s=0$, we obtain (2.1).

Proposition 2.1. Each function $K_{n}$ satisfies, for all $s \in[0, T[$,

$$
S_{n}(s)+\int_{0}^{s} K_{n}(\xi, s-\xi) S_{n}(\xi) d \xi=1,
$$

where $\int_{0}^{s} K_{n}(\xi, s-\xi) S_{n}(\xi) d \xi$ means

$$
\sum_{0 \leqslant \theta \leqslant \tau-T / n} \int_{\theta}^{\theta+T / n} K_{n}(\theta, s-\xi) S_{n}(\xi) d \xi+\int_{\tau}^{s} K_{n}(\tau, s-\xi) S_{n}(\xi) d \xi
$$

whenever $s \in[\tau, \tau+T / n[$.

Conversely, from (2.7) we obtain (2.6) but only for $t \in[0, T / n[$.

We shall see later $(\$ 4)$ that each function $K_{n}(s, t)$ is analytic in $t$. Thus, considering only analytic kernels, we may say that (2.6) and (2.7) are equivalent. This equivalence, however, no longer holds as $n$ goes to infinity (\$5).

Proof. We prove (2.7) implies (2.6) for $t \in[0, T / n[$. (2.7), for $s \in[\tau, \tau+T / n[$, may be decomposed as follows:

$$
S_{n}(s)+\int_{0}^{\tau} K_{n}(\xi, s-\xi) S_{n}(\xi) d \xi+\int_{\tau}^{s} K_{n}(\tau, s-\xi) S_{n}(\xi) d \xi=1
$$

and, by a change of variable in the last integral, $\eta=s-\xi$,

$$
S_{n}(s)+\int_{0}^{\tau} K_{n}(\xi, s-\xi) S_{n}(\xi) d \xi+\int_{0}^{s-\tau} K_{n}(\tau, \eta) S_{n}(s-\eta) d \eta=1 .
$$

Differentiating (2.8) with respect to $s$ and multiplying by $1 / S_{n}(\tau)$, we obtain, since $S_{n}(0)=1$, the equivalent expression

$$
\begin{aligned}
\frac{S_{n}^{\prime}(s)}{S_{n}(\tau)} & +\frac{1}{S_{n}(\tau)} \int_{0}^{\tau} \frac{\partial K_{n}}{\partial t}(\xi, s-\xi) S_{n}(\xi) d \xi \\
& +K_{n}(\tau, s-\tau)+\int_{0}^{s-\tau} K_{n}(\tau, \eta) \frac{S_{n}^{\prime}(s-\eta)}{S_{n}(\tau)} d \eta=0 .
\end{aligned}
$$

Since $s \in\left[\tau, \tau+T / n\right.$ [, we may write $s=\tau+\delta$ with $\delta \in\left[0, T / n\left[\right.\right.$. As $g_{n}(\tau, \delta)=$ $-S_{n}^{\prime}(\tau+\delta) / S_{n}(\tau),(2.9)$ becomes

$$
\begin{aligned}
-g_{n}(\tau, \delta) & +\frac{1}{S_{n}(\tau)} \int_{0}^{\tau} \frac{\partial K_{n}}{\partial t}(\xi, \delta+\tau-\xi) S_{n}(\xi) d \xi \\
& +K_{n}(\tau, \delta)-\int_{0}^{\delta} K_{n}(\tau, \eta) g_{n}(\tau, \delta-\eta) d \eta=0
\end{aligned}
$$

which is precisely (2.6) for $t=\delta$.

The proof of the converse is analogous. 
3. Integral representation of the solution of a convolution equation of type $G-G * g=g$. Let $\beta(x)$ be a real function satisfying

$$
0<\underline{\alpha} \leqslant \beta(x) \leqslant \bar{\alpha}, \quad \forall x \in[0,1],
$$

and $\operatorname{dm}(x)$ a positive measure such that $\int_{0}^{1} d m(x)=1$. Let $g$ be given by

$$
g(t)= \begin{cases}0, & t<0 \\ \int_{0}^{1} \beta(x) e^{-t \beta(x)} d m(x), & t \geqslant 0 .\end{cases}
$$

As we have seen in $\S 1$ the solution of

$$
G-G * g=g,
$$

unique and vanishing outside $[0,+\infty[$, may be given as the sum of the locally convergent series $g+g * g+g * g * g+\cdots$, since $\int_{0}^{+\infty}|g|=1$. We shall represent this same solution as an integral.

Proposition 3.1. The solution $G$ of (3.3) with $g$ given by (3.2) and $\beta$ satisfying (3.1) admits the representation

$$
G(t)=G(\infty)-\int_{-\bar{\alpha}}^{-\underline{\alpha}} \frac{e^{t \lambda}}{\lambda} d \alpha(\lambda), \quad \forall t \in[0,+\infty[,
$$

where $d \alpha$ is uniquely determined as a positive measure with support in $[-\bar{\alpha},-\underline{\alpha}]$, and $G(\infty)=\left[\int_{0}^{1} 1 / \beta(x) d m(x)\right]^{-1}$.

$G$ and $d \alpha$ also satisfy the following estimates:

$$
\begin{gathered}
(-1)^{m} G^{(m)}(t) \geqslant 0, \quad \forall t \in[0,+\infty[, \forall m=0,1, \ldots . \\
\left|G^{(m)}(t)\right| \leqslant \bar{\alpha}^{m+1}, \quad \forall t \in[0,+\infty[, \forall m=0,1, \ldots . \\
\int_{-\bar{\alpha}}^{-\underline{\alpha}} d \alpha(\lambda) \leqslant \bar{\alpha}^{2} .
\end{gathered}
$$

Before proving this proposition we need two lemmas.

Let us consider the Laplace transform $F$ of $D G-G(0) \delta_{0}$, where $D G$ is the derivative, in the distribution sense, of $G$.

$$
\begin{aligned}
& F(p)=\left(D G-G(0) \delta_{0}\right)^{\wedge}=p \hat{G}(p)-G(0)=\frac{p \hat{g}(p)}{1-\hat{g}(p)}-G(0), \\
& \hat{g}(p)=\int_{0}^{1} \frac{\beta(x)}{\beta(x)+p} d m(x),
\end{aligned}
$$

hence

$$
F(p)=\left[\int_{0}^{1} \frac{\beta(x)}{\beta(x)+p} d m(x) / \int_{0}^{1} \frac{1}{\beta(x)+p} d m(x)\right]-g(0) .
$$

Let

$$
J(p)=\left[\int_{0}^{1} \frac{d m(x)}{\beta(x)+p}\right]^{-1}
$$


then

$$
F(p)=-p+J(p)-g(0) .
$$

Let us consider the complex variable function

$$
f(z)=F(z+c)=J(z+c)-(z+c)-g(0),
$$

with $c=-(\bar{\alpha}+\underline{\alpha}) / 2$ and let $M=(\bar{\alpha}-\underline{\alpha}) / 2$.

LEMMA 3.1. The function $f$ given by (3.12) satisfies:

(i) $f$ is analytic outside the interval $[-M, M]$.

(ii) $f(\infty)=0$.

(iii) $\operatorname{Im}(f(z)) \geqslant 0$ if $\operatorname{Im}(z)>0$.

PROOF. (i) Straightforward from definition of $f$.

(ii) We consider

$$
J(z)=1 / \int_{0}^{1} \frac{d m(x)}{\beta(x)+z}=1 / \int_{0}^{1} \frac{1 / z}{\beta(x) / z+1} d m(x)=z / \int_{0}^{1} \frac{d m(x)}{\beta(x) / z+1} .
$$

For $|z|$ large enough we have $|\beta(x) / z|<1, \forall x \in[0,1]$, hence

$$
J(z)=\frac{z}{1-z^{-1} a_{1}-z^{-2} a_{2}-\cdots}, \quad \text { with } a_{n}=(-1)^{n+1} \int_{0}^{1} \beta^{n}(x) d m(x) .
$$

When $z \rightarrow \infty, \lim \left|z^{-1} a_{1}+z^{-2} a_{2}+\cdots\right|=0$; then, for $|z|$ large enough

$$
J(z)=z\left[1+\left(z^{-1} a_{1}+z^{-2} a_{2}+\cdots\right)+\left(z^{-1} a_{1}+z^{-2} a_{2}+\cdots\right)^{2}+\cdots\right],
$$

hence

$$
J(z)+z+\int_{0}^{1} \beta(x) d m(x)+O(1 / z) .
$$

From (3.12) $f(z)=O(1 /(z+c))$ so $f(\infty)=0$.

(iii) $\operatorname{Im} F(z) \geqslant 0$, when $\operatorname{Im}(z)>0$, implies, since $c$ is real, (iii). Let $z=p+i q$. Then $\operatorname{Im} F=\operatorname{Im} J-q$, where

$$
\operatorname{Im} J=q B /\left(A^{2}+q^{2} B^{2}\right),
$$

with

$$
\begin{gathered}
A=\int_{0}^{1} \frac{\beta(x)+p}{[\beta(x)+p]^{2}+q^{2}} d m(x), \quad B=\int_{0}^{1} \frac{d m(x)}{[\beta(x)+p]^{2}+q^{2}} . \\
\operatorname{Im} F=q B /\left(A^{2}+q^{2} B^{2}\right)-q \geqslant 0, \quad \text { when } q>0,
\end{gathered}
$$

is equivalent to $B /\left(A^{2}+q^{2} B^{2}\right) \geqslant 1$, which is the same as $B\left(1-q^{2} B\right) \geqslant A^{2}$ or, replacing $A$ and $B$ by their values:

$$
\begin{aligned}
\left(\int_{0}^{1} \frac{\beta(x)+p}{[\beta(x)+p]^{2}+q^{2}}\right. & d m(x))^{2} \\
& \leqslant \int_{0}^{1} \frac{[\beta(x)+p]^{2}}{[\beta(x)+p]^{2}+q^{2}} d m(x) \int_{0}^{1} \frac{d m(x)}{[\beta(x)+p]^{2}+q^{2}},
\end{aligned}
$$

which is true by the Cauchy-Schwarz inequality. 
LEMMA 3.2. There exists a positive measure $d \alpha$ with support in $[-\bar{\alpha},-\underline{\alpha}]$, where $\underline{\alpha}$ and $\bar{\alpha}$ are, respectively, the lower and upper bounds of $\beta[c f .(3.1)]$, such that the Laplace transform $F$ of $D G-G(0) \delta_{0}$ admits the representation

$$
F(z)=\int_{-\bar{\alpha}}^{-\underline{\alpha}} \frac{1}{\lambda-z} d \alpha(\lambda), \quad \forall z: \operatorname{Re} z>-\underline{\alpha} .
$$

Proof. We use a result of Korányi [2] which guarantees that for a function $f$ satisfying (i)-(iii) of Lemma 3.1, there exists a nondecreasing bounded function $\alpha(t)$, constant outside the interval $[-M, M]$, such that

$$
f(z)=\int_{-M}^{M} \frac{1}{t+z} d \alpha(t), \quad \forall z:|z|>M .
$$

As $F$ is related to $f$ by (3.12), for $|z-c|>M$,

$$
F(z)=\int_{-M}^{M} \frac{1}{(t-c)-z} d \alpha(t)
$$

and, by the change of variable $t=(\lambda-c)=\phi(\lambda)$,

$$
F(z)=\int_{-M+c}^{M+c} \frac{1}{\lambda-z} d \alpha(\phi(\lambda)) .
$$

Finally, writing $\alpha(\lambda)$ instead of $\alpha(\phi(\lambda))$, and by the definitions of $M$ and $c$, we obtain (3.14).

Proof of Proposition 3.1. From the uniqueness of the Laplace transform and Lemma 3.2, we conclude that

$$
G^{\prime}=D G-G(0) \delta_{0}=-\int_{-\bar{\alpha}}^{-\underline{\alpha}} e^{t \lambda} d \alpha(\lambda), \quad \forall t \geqslant 0 .
$$

Integrating (3.16) we obtain (3.4), whose uniqueness stems from the fact that the measure $d \alpha$ is completely determined by its values over all the polynomials $\lambda^{m}$, $\forall m \geqslant 0$ : as a matter of fact, from (3.4),

$$
\begin{aligned}
G(t)-G(\infty) & =\sum_{m \geqslant 1} G^{(m)}(0) \frac{t^{m}}{m !}, \quad \text { with } G^{(m)}(0) \\
& =\int_{-\bar{\alpha}}^{-\underline{\alpha}} \lambda^{m-1} d \alpha(\lambda), \quad \forall m \geqslant 1
\end{aligned}
$$

In particular,

$$
G(0)=G(\infty)-\int_{-\bar{\alpha}}^{-\underline{\alpha}} \frac{1}{\lambda} d \alpha(\lambda) .
$$

Equation (3.3) yields $G(0)=g(0)=\int_{0}^{1} \beta(x) d m(x)$. On the other hand $F(0)$ can be represented in two different ways, from (3.9) and (3.14), respectively:

$$
F(0)=\frac{1}{\int_{0}^{1} d m(x) / \beta(x)}-G(0)=\int_{-\bar{\alpha}}^{-\underline{\alpha}} \frac{1}{\lambda} d \alpha(\lambda),
$$

which, because of (3.17), gives $G(\infty)=\left[\int_{0}^{1} 1 / \beta(x) d m(x)\right]^{-1}$. 
By differentiating (3.3) at zero several times we obtain

$$
\left\{\begin{array}{l}
G(0)=a_{1}, \\
G^{\prime}(0)=a_{2}+a_{1}^{2}, \\
\vdots \\
G^{(m)}(0)=a_{m+1}+a_{m} G(0)+\cdots+a_{m-p} G^{(p)}(0)+\cdots+a_{1} G^{(m-1)}(0),
\end{array} .\right.
$$

where

$$
a_{m}=(-1)^{m+1} \int_{0}^{1} \beta^{m}(x) d m(x)=g^{(m-1)}(0) .
$$

In particular, the total variation $V(\alpha)$ of $\alpha$ in $[-\bar{\alpha},-\underline{\alpha}]$ is given by

$$
V(\alpha)=\int_{-\bar{\alpha}}^{-\underline{\alpha}} d \alpha(\lambda)=G^{\prime}(0)=\int_{0}^{1} \beta^{2}(x) d m(x)-\left[\int_{0}^{1} \beta(x) d m(x)\right]^{2} \leqslant \bar{\alpha}^{2} .
$$

Finally, differentiating (3.4) we get

$$
G^{(m)}(t)=-\int_{-\bar{\alpha}}^{-\underline{\alpha}} \lambda^{m-1} e^{i \lambda} d \alpha(\lambda), \quad \forall m \geqslant 1,
$$

from which (3.5) and (3.6) are easily derived:

$$
\left|G^{(m)}(t)\right| \leqslant \bar{\alpha}^{m-1} V(\alpha) \leqslant \bar{\alpha}^{m-1} \bar{\alpha}^{2}=\bar{\alpha}^{m+1}, \quad \forall m \geqslant 1 .
$$

4. Estimates for kernels $K_{n}(s, t)$ and their derivatives with respect to $t$. In this section we always consider the same partition of the interval $[0, T$ [ into $n$ equal subintervals. To simplify notation we eliminate the index $n$ in $K_{n}(s, t)$ and represent by $K_{\tau}(t)$ the restriction of $K_{n}$ to $[\tau, \tau+\Delta[\times[0,+\infty$, with $\Delta=T / n$. We define $G_{\tau}(t)$ and $P_{\tau}(t)$ in a similar way.

In Proposition 3.1 we proved (3.6); sometimes, however, we shall make use of a weaker result:

$$
\left|G_{\tau}^{(m)}(t)\right| \leqslant 2^{m} \bar{\alpha}^{m+1}, \quad \forall m \geqslant 0, \forall t \in[0,+\infty[.
$$

By differentiating (2.2) several times we obtain

$$
\begin{aligned}
& K_{\tau}=P_{\tau}+\left(P_{\tau} * G_{\tau}\right)+G_{\tau}, \\
& K_{\tau}^{\prime}=P_{\tau}^{\prime}+P_{\tau} G_{\tau}(0)+\left(P_{\tau} * G_{\tau}^{\prime}\right)+G_{\tau}^{\prime}, \\
& \vdots \\
& K_{\tau}^{(m)}=P_{\tau}^{(m)}+P_{\tau}^{(m-1)} G_{\tau}(0)+\cdots+P_{\tau} G_{\tau}^{(m-1)}(0)+\left(P_{\tau} * G_{\tau}^{(m)}\right)+G_{\tau}^{(m)} .
\end{aligned}
$$

Proposition 4.1. Let $|\cdot|$ be the $L^{\infty}(0,+\infty)$ norm. Formally we have the following inequalities:

$$
\begin{gathered}
\left|P_{\tau} * G_{\tau}^{(m)}\right| \leqslant\left|P_{\tau}\right| \bar{\alpha}^{m}, \quad m=1,2, \ldots, \\
\left|P_{\tau}^{(m)}\right| \leqslant c \cdot \Delta \sum_{0 \leqslant \theta<\tau}\left|K_{\theta}^{(m+1)}\right|, \quad \text { with } c=e^{\bar{\alpha} T}, m=0,1,2, \ldots, \\
\left|K_{\tau}^{(m)}\right| \leqslant\left|P_{\tau}^{(m)}\right|+\left|P_{\tau}^{(m-1)}\right| \bar{\alpha}+\cdots+\left|P_{\tau}\right| \bar{\alpha}^{m}+\left|P_{\tau}\right| \bar{\alpha}^{m}+2^{m} \bar{\alpha}^{m+1}, \\
m=1,2, \ldots
\end{gathered}
$$


Proof. For $m \geqslant 1$ we have

$$
\begin{aligned}
\left|P_{\tau} * G_{\tau}^{(m)}\right| & =\left|\int_{0}^{t} P_{\tau}(t-s) G_{\tau}^{(m)}(s) d s\right| \leqslant \int_{0}^{t}\left|P_{\tau}(t-s)\right| \cdot\left|G_{\tau}^{(m)}(s)\right| d s \\
& \leqslant\left|P_{\tau}\right| \int_{0}^{t}\left|G_{\tau}^{(m)}(s)\right| d s .
\end{aligned}
$$

If $m$ is even, $G_{\tau}^{(m)} \geqslant 0$ and $G_{\tau}^{(m-1)} \leqslant 0$, we have

$$
\begin{aligned}
\int_{0}^{t}\left|G_{\tau}^{(m)}(s)\right| d s & =\int_{0}^{t} G_{\tau}^{(m)}(s) d s=G_{\tau}^{(m-1)}(t)-G_{\tau}^{(m-1)}(0) \\
& =\left|G_{\tau}^{(m-1)}(0)\right|-\left|G_{\tau}^{(m-1)}(t)\right| ;
\end{aligned}
$$

thus

$$
\int_{0}^{t}\left|G_{\tau}^{(m)}(s)\right| d s \leqslant\left|G_{\tau}^{(m-1)}(0)\right| \leqslant \bar{\alpha}^{m}
$$

which proves (4.3).

If $m$ is odd, $G_{\tau}^{(m)} \leqslant 0$ and $G_{\tau}^{(m-1)} \geqslant 0$, we obtain the same estimate.

On the other hand, for any $m \geqslant 0$, we have

$$
P_{\tau}^{(m)}(t)=-\frac{1}{S_{n}(\tau)} \sum_{0 \leqslant \theta<\tau} \int_{\theta}^{\theta+\Delta} K_{\theta}^{(m+1)}(t+\tau-s) S_{n}(s) d s .
$$

Then, since $\left|S_{n}(s)\right| \leqslant 1 \forall s \in[0, T[$ and

$$
S_{n}(\tau)=\int_{0}^{1} I\left(\beta_{n}, \tau\right) d x>\int_{0}^{1} I\left(\beta_{n}, T\right) d x>e^{-\bar{\alpha} T}=1 / c,
$$

we obtain (4.4). Finally, from (3.6) and (4.1)-(4.3), we deduce (4.5).

Proposition 4.2. For $m \geqslant 1$ the following estimate occurs:

$$
\left|K_{\theta_{i}}^{(m)}\right| \leqslant 2^{m} \bar{\alpha}^{m+1}(1+4 \bar{\alpha} c \Delta)^{i},
$$

with $\theta_{i}=i \Delta, i=0,1, \ldots, n-1, n$ being the number of subintervals of the partition, $\Delta=T / n$ and $m=1,2, \ldots$ representing the order of differentiation.

Proof. We shall prove (4.6) by induction over the indices $i$ of the partition: If $i=0, K_{\theta_{i}}$ is the solution of (3.3), thus, using (4.1), $\left|K_{\theta_{0}}^{(m)}\right| \leqslant 2^{m} \bar{\alpha}^{m+1}$.

Let us admit that

$$
\left|K_{\theta_{j}}^{(m)}\right| \leqslant\left(2^{m} \bar{\alpha}^{m+1}\right)(1+4 \bar{\alpha} c \Delta)^{j}, \quad \forall j: 0 \leqslant j \leqslant i-1 .
$$

We must prove

$$
\left|K_{\theta_{i}}^{(m)}\right| \leqslant 2^{m} \bar{\alpha}^{m+1}(1+4 \bar{\alpha} c \Delta)^{i} .
$$

From (4.4) and (4.5) we deduce

$$
\left|K_{\theta_{i}}^{(m)}\right| \leqslant c \Delta \sum_{0 \leqslant j \leqslant i-1}\left[\left|K_{\theta_{j}}^{(m+1)}\right|+\left|K_{\theta_{j}}^{(m)}\right| \bar{\alpha}+\cdots+2\left|K_{\theta_{j}}^{\prime}\right| \bar{\alpha}^{m}\right]+2^{m} \bar{\alpha}^{m+1} .
$$

Using the induction hypothesis,

$$
\begin{aligned}
\left|K_{\theta_{i}}^{(m)}\right| \leqslant & c \Delta \sum_{0 \leqslant j \leqslant i-1}\left[2^{m+1} \bar{\alpha}^{m+2}(1+4 \bar{\alpha} c \Delta)^{j}+\cdots+2 \cdot 2 \cdot \bar{\alpha}^{2} \cdot \bar{\alpha}^{m}(1+4 \bar{\alpha} c \Delta)^{j}\right] \\
& +2^{m} \bar{\alpha}^{m+1}
\end{aligned}
$$


or, which is the same,

$$
\left|K_{\theta_{i}}^{(m)}\right| \leqslant c \Delta\left[\sum_{0 \leqslant j \leqslant i-1}(1+4 \bar{\alpha} c \Delta)^{j}\right] \bar{\alpha}^{m+2}\left(2^{m+1}+2^{m}+\cdots+2+2\right)+2^{m} \overline{\boldsymbol{\alpha}}^{m+1} .
$$

Using twice the formula of the sum of the first terms of a geometric sequence we may write

$$
\left|K_{\theta_{i}}^{(m)}\right| \leqslant c \Delta\left[\frac{1-(1+4 \bar{\alpha} c \Delta)^{i}}{1-(1+4 \bar{\alpha} c \Delta)}\right] \bar{\alpha}^{m+2} 2^{m+2}+2^{m} \bar{\alpha}^{m+1}
$$

and, finally,

$$
\left|K_{\theta_{i}}^{(m)}\right| \leqslant 2^{m} \bar{\alpha}^{m+1}(1+4 \bar{\alpha} c \Delta)^{i} .
$$

For $m=0$ we also obtain a similar estimate by induction.

Proposition 4.3. Let $|\cdot|$ be the $L^{\infty}(0,+\infty)$ norm. Formally, we have the following inequalities:

$$
\left|P_{\tau} * G_{\tau}\right| \leqslant c^{\prime}\left|P_{\tau}\right|+G_{\tau}(\infty)\left|\int_{0}^{t} P_{\tau}(s) d s\right|
$$

where $c^{\prime}$ satisfies

$$
0<c^{\prime}=\int_{-\bar{\alpha}}^{-\underline{\alpha}} \frac{1}{\lambda^{2}} d \alpha_{\tau}(\lambda) \leqslant \frac{\bar{\alpha}^{2}-\underline{\alpha}^{2}}{\underline{\alpha}^{2}}
$$

and

$$
\left|K_{\tau}\right| \leqslant 2 \bar{c} \Delta\left[\sum_{0 \leqslant \theta<\tau}\left|K_{\theta}^{\prime}\right|+\bar{\alpha} \sum_{0 \leqslant \theta<\tau}\left|K_{\theta}\right|\right]+\bar{\alpha}
$$

where $\bar{c}=\max \left\{c, c c^{\prime}\right\}$.

Proof. Consider the function $H_{\tau}(t)=G_{\tau}(t)-G_{\tau}(\infty) \geqslant 0$. Thus

$$
\begin{gathered}
P_{\tau} * G_{\tau}=P_{\tau} * H_{\tau}+G_{\tau}(\infty) \int_{0}^{t} P_{\tau}(s) d s, \\
\left|P_{\tau} * G_{\tau}\right| \leqslant\left|P_{\tau}\right| \int_{0}^{t} H_{\tau}(s) d s+G_{\tau}(\infty)\left|\int_{0}^{t} P_{\tau}(s) d s\right| .
\end{gathered}
$$

From Proposition 3.1

$$
\int_{0}^{t} H_{\tau}(s) d s=\int_{-\bar{\alpha}}^{-\underline{\alpha}} \frac{1}{\lambda^{2}} d \alpha_{\tau}(\lambda)-\int_{-\bar{\alpha}}^{-\underline{\alpha}} \frac{e^{t \lambda}}{\lambda^{2}} d \alpha_{\tau}(\lambda) \leqslant \int_{-\bar{\alpha}}^{-\underline{\alpha}} \frac{1}{\lambda^{2}} d \alpha_{\tau}(\lambda)=c^{\prime},
$$

which proves (4.7).

(4.8) is easily obtained, considering (4.2), (3.6), (4.4), (4.7) and the fact that

$$
\int_{0}^{t} P_{\tau}(s) d s=-\frac{1}{S_{n}(\tau)} \sum_{0 \leqslant \theta<\tau} \int_{\theta}^{\theta+\Delta}\left[K_{\theta}(t+\tau-s)-K_{\theta}(\tau-s)\right] S_{n}(s) d s
$$

satisfies

$$
\left|\int_{0}^{t} P_{\tau}(s) d s\right| \leqslant 2 c \Delta \sum_{0 \leqslant \theta<\tau}\left|K_{\theta}\right|
$$


Proposition 4.4. The following estimate occurs:

$$
\left|K_{\theta_{i}}\right| \leqslant \bar{\alpha}(1+6 \bar{\alpha} c \bar{\alpha} \Delta)^{i}
$$

with the same notation for the indices used in Proposition 4.2.

Proof. The proof is basically the same as for Proposition 4.2, replacing inequality (4.6) by (4.8) and using, to estimate the first order derivative of $K_{\theta_{j}}$,

$$
\left|K_{\theta_{j}}^{\prime}\right| \leqslant 2 \bar{\alpha}^{2}(1+6 \bar{\alpha} \bar{c} \Delta)^{j}
$$

instead of (4.6).

From Propositions 4.2 and 4.4 we easily obtain the following result:

Proposition 4.5. For any partition of the interval $[0, T[$ into $n$ equal subintervals, the following inequalities hold for any $(s, t) \in[0, T[\times[0,+\infty[$ and any $m \geqslant 0$ :

$$
\left|\partial^{m} K_{n}(s, t) / \partial t^{m}\right| \leqslant 2^{m} \bar{\alpha}^{m+1} e^{C(\underline{\alpha}, \bar{\alpha}, T) \cdot T}
$$

$C(\underline{\alpha}, \bar{\alpha}, T)$ being a positive real value depending only on the bounds of $\beta(c f .(1.1))$ and $T$.

As a consequence of (4.11), $K$ is analytic in $t \in[0,+\infty[$ for each $s \in[0, T[$ :

$$
K_{n}(s, t)=\sum_{m \geqslant 0} \phi_{m, n}(s) \frac{t^{m}}{m !},
$$

where

$$
\phi_{m, n}(s)=\partial^{m} K_{n}(s, 0) / \partial t^{m}
$$

Proof. From Propositions 4.2 and 4.4 we have, for all $m, n \geqslant 0$ and for all $(s, t) \in[0, T[\times[0,+\infty[$,

$$
\left|\partial^{m} K_{n}(s, t) / \partial t^{m}\right| \leqslant 2^{m} \bar{\alpha}^{m+1}\left(1+6 \bar{\alpha} \bar{c} \frac{T}{n}\right)^{n} \leqslant 2^{m} \bar{\alpha}^{m+1} e^{6 \bar{\alpha} \bar{c} T},
$$

since the sequence $(1+6 \bar{\alpha} \bar{c} T / n)^{n}$ increases towards $e^{6 \bar{\alpha} \bar{c} T}$.

\section{Proof of Theorem 1.1.}

LEMMA 5.1. To each natural $m$ the functions $\phi_{m, n}, n \in \mathbf{N}$, defined in $[0, T[$ by (4.12), have their total variation uniformly bounded.

Proof. As we are dealing with step functions, to estimate the total variation $V_{0}^{T}\left(\phi_{m, n}\right)$ of $\phi_{m, n}$, it is enough to take the partitions of [0,T[ such that $\phi_{m, n}$ are constant in each subinterval. 
Let $[\tau, \tau+\Delta[, \Delta=T / n$, be any subinterval of the partition. We keep the notation of $\S 4$. Using (4.2) we have

$$
\begin{aligned}
& \left|\phi_{m, n}(\tau)-\phi_{m, n}(\tau+\Delta)\right|=\left|K_{\tau}^{(m)}(0)-K_{\tau+\Delta}^{(m)}(0)\right| \leqslant\left|P_{\tau}^{(m)}(0)-P_{\tau+\Delta}^{(m)}(0)\right| \\
& \quad+\sum_{j=1}^{m}\left|P_{\tau}^{(m-j)}(0) G_{\tau}^{(j-1)}(0)-P_{\tau+\Delta}^{(m-j)} G_{\tau+\Delta}^{(j-1)}(0)\right|+\left|G_{\tau}^{(m)}(0)-G_{\tau+\Delta}^{(m)}(0)\right| \\
& \leqslant\left|P_{\tau}^{(m)}(0)-P_{\tau+\Delta}^{(m)}(0)\right|+\left|G_{\tau}^{(m)}(0)-G_{\tau+\Delta}^{(m)}(0)\right| \\
& \quad+\sum_{j=1}^{m}\left|P_{\tau+\Delta}^{(m-j)}(0)\right| \cdot\left|G_{\tau}^{(j-1)}(0)-G_{\tau+\Delta}^{(j-1)}(0)\right| \\
& \quad+\sum_{j=1}^{m}\left|G_{\tau}^{(j-1)}(0)\right| \cdot\left|P_{\tau}^{(m-j)}(0)-P_{\tau+\Delta}^{(m-j)}(0)\right| .
\end{aligned}
$$

From the definition of $P_{\tau}$, and using (4.11),

$$
\left|P_{\tau}^{(j)}(0)\right| \leqslant c T \bar{\alpha} e^{C(\underline{\alpha}, \bar{\alpha}, T) \cdot T}(2 \bar{\alpha})^{j},
$$

and

$$
\begin{aligned}
\mid P_{\tau}^{(j)}(0) & -P_{\tau+\Delta}^{(j)}(0) \mid \\
\leqslant & \frac{1}{S_{n}(\tau+\Delta)} \sum_{0 \leqslant \theta<\tau} \int_{\theta}^{\theta+\Delta}\left|K_{\theta}^{(j+1)}(\tau+\Delta-s)-K_{\theta}^{(j+1)}(\tau-s)\right| S_{n}(s) d s \\
& +\left|\frac{1}{S_{n}(\tau+\Delta)}-\frac{1}{S_{n}(\tau)}\right| \cdot \sum_{0 \leqslant \theta<\tau} \int_{\theta}^{\theta+\Delta}\left|K_{\theta}^{(j+1)}(\tau-s)\right| S_{n}(s) d s \\
& +\frac{1}{S_{n}(\tau+\Delta)} \int_{\tau}^{\tau+\Delta}\left|K_{\tau}^{(j+1)}(\tau+\Delta-s)\right| S_{n}(s) d s .
\end{aligned}
$$

Using the mean value theorem and (4.11),

$$
\begin{aligned}
\left|P_{\tau}^{(j)}(0)-P_{\tau+\Delta}^{(j)}(0)\right| \leqslant & c \sum_{0 \leqslant \theta<\tau} \int_{\theta}^{\theta+\Delta} \max _{t}\left|K_{\theta}^{(j+2)}(t)\right| \Delta d s \\
& +c^{2} \int_{0}^{1}\left|I\left(\beta_{n}, \tau+\Delta\right)-I\left(\beta_{n}, \tau\right)\right| d s \sum_{0 \leqslant \theta<\tau} \Delta \max _{t}\left|K_{\theta}^{(j+1)}(t)\right| \\
& +c \Delta \max _{t}\left|K_{\tau}^{(j+1)}(t)\right|\left|P_{\tau}^{(j)}(0)-P_{\tau+\Delta}^{(j)}(0)\right| \\
\leqslant & c 2^{j+2} \bar{\alpha}^{j+3} e^{c T} \Delta+c^{2} \bar{\alpha} \Delta 2^{j+1} \bar{\alpha}^{j+2} e^{c T}+c \Delta 2^{j+1} \bar{\alpha}^{j+2} e^{C T} \\
= & c(j) / n,
\end{aligned}
$$

where $c(j)$ represents some constant depending on $j$.

By Proposition 3.1 we have

$$
\left|G_{\tau}^{(j)}(0)\right| \leqslant \bar{\alpha}^{j+1}
$$


Adding the $\left|\phi_{m, n}(\tau)-\phi_{m, n}(\tau+\Delta)\right|$, with $\tau=0, \ldots,(n-1) \Delta$, and using the second inequality of (5.1), (5.3) and (5.4), we obtain

$$
\begin{aligned}
& \sum_{\tau}\left|\phi_{m, n}(\tau)-\phi_{m, n}(\tau+\Delta)\right| \leqslant c(m)+\sum_{\tau}\left|G_{\tau}^{(m)}(0)-G_{\tau+\Delta}^{(m)}(0)\right| \\
& \quad+\sum_{j=1}^{m} c(m-j) \cdot \sum_{\tau}\left|G_{\tau}^{(j-1)}(0)-G_{\tau+\Delta}^{(j-1)}(0)\right|+\sum_{j=1}^{m} \bar{\alpha}^{j} c(m-j) .
\end{aligned}
$$

Finally, to estimate (5.5) independently of $n$, it is enough to show that

$$
\sum_{\tau}\left|G_{\tau}^{(j)}(0)-G_{\tau+\Delta}^{(j)}(0)\right|
$$

may be estimated independently of $n$ for each $j \geqslant 0$. We shall prove this by induction.

For each $j \geqslant 0$ we have, from (3.18) and

$$
a_{p}(\tau)=(-1)^{p+1} \int_{0}^{1} \beta^{p}(x, \tau) d m_{\tau}(x) \quad(\mathrm{cf} .(2.3)),
$$

the following estimates:

$$
\begin{aligned}
\left|G_{\tau}^{(j)}(0)-G_{\tau+\Delta}^{(j)}(0)\right| & \\
= & \left|a_{j+1}(\tau)+\sum_{i=1}^{j} a_{i}(\tau) G_{\tau}^{(j-i)}(0)-a_{j+1}(\tau+\Delta)-\sum_{i=1}^{j} a_{i}(\tau+\Delta) G_{\tau+\Delta}^{(j-i)}(0)\right| \\
\leqslant & \left|a_{j+1}(\tau)-a_{j+1}(\tau+\Delta)\right|+\sum_{i=1}^{j}\left|a_{i}(\tau+\Delta)\right| \cdot\left|G_{\tau}^{(j-i)}(0)-G_{\tau+\Delta}^{(j-i)}(0)\right| \\
& +\sum_{i=1}^{j}\left|a_{i}(\tau)-a_{i}(\tau+\Delta)\right| \cdot\left|G_{\tau}^{(j-i)}(0)\right|,
\end{aligned}
$$

which implies

$$
\begin{aligned}
\sum_{\tau}\left|G_{\tau}^{(j)}(0)-G_{\tau+\Delta}^{(j)}(0)\right| \leqslant & \sum_{\tau}\left|a_{j+1}(\tau)-a_{j+1}(\tau+\Delta)\right| \\
& +\sum_{i=1}^{j} \bar{\alpha}^{i} \sum_{\tau}\left|G_{\tau}^{(j-i)}(0)-G_{\tau+\Delta}^{(j-i)}(0)\right| \\
& +\sum_{i=1}^{j} \bar{\alpha}^{m-i+1} \sum_{\tau}\left|a_{i}(\tau+\Delta)-a_{i}(\tau)\right| .
\end{aligned}
$$

The proof by induction is straightforward from (5.9), since, for each $j \geqslant 0$, we easily obtain the following estimates:

$$
\sum_{\tau}\left|a_{j}(\tau)-a_{j}(\tau+\Delta)\right| \leqslant c(j)+\sum_{\tau} \int_{0}^{1}\left|\beta^{j}(x, \tau+\Delta)-\beta^{j}(x, \tau)\right| d x,
$$

where the last sum is uniformly bounded because of hypothesis (1.1)(ii) concerning the partial derivative of $\beta$. 
COROLlaRY. There exists a subsequence $\phi_{m, n_{h}}$ of $\phi_{m, n}$ and functions $\phi_{m}$ defined on $[0, T[$ such that for all $m \geqslant 0$ and $s \in[0, T[$,

$$
\lim _{k \rightarrow+\infty} \phi_{m, n_{k}}(s)=\phi_{m}(s) \text {. }
$$

Proof. Since $\phi_{m, n}$ are uniformly bounded in $n$ (cf. Proposition 4.5) and, by Lemma 5.1, they have uniformly bounded total variation, we can use Helly's theorems [1], for each $m$ and for a suitable subset of functions $\phi_{m, n}$, and we finally consider a diagonal subsequence.

Proposition 5.1 (Local ExistenCE). Let $\beta$ be a function satisfying the hypothesis of Theorem 1.1, approached by the step functions sequence $\beta_{n}$, and let $K_{n}$ be the corresponding kernels as defined in $\$ 2$. We claim that $K_{n}$ converges pointwise to a function $K$, defined for $(s, t) \in[0, T[\times[0,+\infty[$, vanishing for $t<0$, of the form

$$
K(s, t)=\sum_{m \geqslant 0} \phi_{m}(s) \frac{t^{m}}{m !}, \quad \phi_{m}(s)=\frac{\partial^{m} K}{\partial t^{m}}(s, 0),
$$

satisfying (4.11) and (1.8) for all $(s, t) \in[0, T[\times[0,+\infty[$.

Proof. Let $\phi_{m}(s)$ of $(5.10)$ be the limit function obtained in the corollary of Lemma 5.1. The function $K$ defined by (5.10) is then the pointwise limit of the sequence given by

$$
K_{n_{k}}(s, t)=\sum_{m \geqslant 0} \phi_{m, n_{k}}(s) \frac{t^{m}}{m !}
$$

which clearly satisfies estimates (4.11).

Letting $n \rightarrow \infty$ in (2.6), we obtain functions $g, S$ and $P$ as the pointwise limits of $g_{n}, S_{n}$ and $P_{n_{k}}$, respectively. Thus, as $n \rightarrow \infty$ and by Lebesgue's theorem, (2.6) becomes (1.8).

Proposition 5.2 (Local UNIQUeness). Let $\beta$ be a function satisfying the hypothesis of Theorem 1.1. Then there exists only one function $K(s, t)$, defined in $[0, T[\times \mathbf{R}$, vanishing for $t<0$, analytic with respect to $t \in[0,+\infty[$ (i.e. of the form (5.10)) and satisfying (1.8) for all $(s, t) \in[0, T[\times[0,+\infty[$.

Proof. By differentiating (1.8) several times with respect to $t$ and writing $K_{t}^{(p)}$ for $\partial^{p} K / \partial t^{p}$, we obtain

$$
\begin{aligned}
& K_{t}^{(p)}(s, t)-\left[K_{t}^{(p)}(s, \cdot) * g(s, \cdot)\right](t) \\
& =\frac{-1}{S(s)} \int_{0}^{s} K_{t}^{(p+1)}(\xi, t+s-\xi) S(\xi) d \xi \\
& \quad+\sum_{i=1}^{p} K_{t}^{(p-i)}(s, 0) g_{t}^{(i-1)}(s, t)+g_{t}^{(p)}(s, t), \quad \forall p \geqslant 0
\end{aligned}
$$


(We understand that the sum is zero if $p=0$.) Making $t=0$ in (5.11) we get an infinite system

$$
\begin{aligned}
K_{t}^{(p)}(s, 0)= & \frac{-1}{S(s)} \int_{0}^{s} K_{t}^{(p+1)}(\xi, s-\xi) S(\xi) d \xi \\
& +\sum_{i=1}^{p} K_{t}^{(p-i)}(s, 0) g_{t}^{(i-1)}(s, 0)+g_{t}^{(p)}(s, 0), \quad \forall p \geqslant 0
\end{aligned}
$$

Let us represent $g^{(p-1)}(s, 0)$, by

$$
\left\{\begin{array}{l}
a_{p}(s)=(-1)^{p+1} \int_{0}^{1} \beta^{p}(x, s) d m_{s}(x), \quad p \geqslant 1 \\
a_{0}(s)=-1
\end{array}\right.
$$

Since any solution of (1.8) is taken with the form (5.10), the uniqueness of $K$ is equivalent to the uniqueness of the functions $\phi_{m}(s)$ with $m \geqslant 0$.

Replacing (5.10) in (5.12) and setting $\psi_{m}(s)=\phi_{m}(s) \cdot S(s)$, we obtain the following infinite system:

$$
\begin{aligned}
\sum_{i=0}^{p} \psi_{p-i}(s) & a_{i}(s)+a_{p+1}(s) S(s) \\
& =\sum_{m \geqslant 0} \int_{0}^{s} \psi_{m+p+1}(\xi) \frac{(s-\xi)^{m}}{m !} d \xi, \quad \forall p \geqslant 0 .
\end{aligned}
$$

Making $p=0$ in (5.14) and differentiating, we obtain

$$
-\psi_{0}^{\prime}(s)+\left[a_{1}(s) S(s)\right]^{\prime}=\psi_{1}(s)+\sum_{m \geqslant 0} \int_{0}^{s} \psi_{m+2}(\xi) \frac{(s-\xi)^{m}}{m !} d \xi .
$$

Finally replacing the sum by its value given by $(5.14)$, for $p=1$, we obtain

$$
-\psi_{0}^{\prime}(s)+\left[a_{1}(s) S(s)\right]^{\prime}=\psi_{0}(s) a_{1}(s)+a_{2}(s) S(s),
$$

with the initial data

$$
\psi_{0}(0)=a_{1}(0)=\int_{0}^{1} \beta(x, 0) d x .
$$

Thus, $\psi_{0}(s)$ is uniquely determined by (5.14).

Let us admit that $\psi_{0}, \ldots, \psi_{p-1}$ are uniquely determined by (5.14). By a similar method we obtain $\psi_{p}$ as the solution of the differential equation

$$
\begin{aligned}
-\psi_{p}^{\prime}(s) & +\left[\sum_{i=1}^{p} \psi_{p-i}(s) a_{i}(s)\right]^{\prime}+\left[a_{p+1}(s) S(s)\right]^{\prime} \\
& =\psi_{p}(s) a_{1}(s)+\sum_{i=2}^{p+1} \psi_{p+1-i}(s) a_{i}(s)+a_{p+2}(s) S(s)
\end{aligned}
$$

with initial data

$$
\psi_{p}(0)=\sum_{i=1}^{p} \psi_{p-i}(0) a_{i}(0)+a_{p+1}(0)
$$


The uniqueness of $K(s, t)$ in $[0, T[\times[0,+\infty[$ for any $T \in[0,+\infty[$, satisfying (1.8), allows us to extend it to $[0,+\infty[\times[0,+\infty[$, and (1.12) holds.

Since $S(0)=1,(1.7)$ is equivalent to the one we obtain by differentiation with respect to $t$ :

$$
K(t, 0)=\frac{-1}{S(t)} \int_{0}^{t} \frac{\partial K}{\partial t}(s, t-s) S(s) d s-\frac{S^{\prime}(t)}{S(t)},
$$

which, since $-S^{\prime}(t) / S(t)=g(t, 0)$, is precisely the first equation of the infinite system (5.14). So we may say that the unique analytic solution, with support in $[0,+\infty[\times[0,+\infty[$, of (1.8) also verifies (1.7), which completes the proof of Theorem 1.1.

Note. With a simple example we are going to show that, in general, (1.7) does not guarantee the uniqueness of the kernel $K$ : there exists an infinity of functions, with support in $[0,+\infty[\times[0,+\infty[$, of the form (5.10) satisfying (1.7).

Suppose $K$ and $L$ are two kernels satisfying (1.7) with difference

$$
(K-L)(s, t)=\sum_{m \geqslant 0} \phi_{m}(s) \frac{t^{m}}{m !}, \quad \forall(s, t) \in[0,+\infty[\times[0,+\infty[,
$$

and satisfying, with $\psi_{m}(s)=\phi_{m}(s) S(s)$,

$$
\sum_{m \geqslant 0} \int_{0}^{t} \psi_{m}(s) \frac{(t-s)^{m}}{m !} d s=0 .
$$

Let $\psi_{m}(s)=f(s), \forall m \geqslant 1$, with $f(0)=0$ and $f \not 0$.

Let

$$
\Phi(t)=\sum_{m \geqslant 1} \int_{0}^{t} \psi_{m}(s) \frac{(t-s)^{m}}{m !} d s=\int_{0}^{t} f(s)\left[e^{(t-s)}-1\right] d s .
$$

We have $\Phi(0)=0$ and $\Phi^{\prime}=\int_{0}^{t} f(s) e^{(t-s)} d s$, so $\Phi^{\prime}(0)=0$.

Finally, let $\psi_{0}(s)=-\Phi^{\prime}(s)$. (5.16) is then satisfied by a sequence of nonzero functions vanishing at the origin.

\section{REFERENCES}

1. A. Kolmogorov and S. Fomin, Eléments de la théorie des fonctions et de l'analyse fonctionnelle, "Mir", Moscow, 1974.

2. A. Korányi, Note on the theory of monotone operator functions, Acta Sci. Math. 16 (1955), 241-245.

3. E. Sanchez-Palencia, Non-homogeneous media and vibration theory, Lecture Notes in Physics, Vol. 127. Springer-Verlag, Berlin and New York, 1980.

Centro de Matemática e Apliçóes Fundamentais, Av. Prof. Gama Pinto 2,1699-Lisboa Codex, PORTUGAL 